\title{
Role of MicroRNA-134-5p in Metabolic Syndrome-Associated Pulmonary Hypertension in Heart Failure with Preserved Ejection Fraction
}

Aidan Hannon ${ }^{1}$, Jia-Rong Jheng ${ }^{2}$, Gunner Halliday², Yen-Chun Lai ${ }^{2}$

${ }^{1}$ Department of Medical Education, Indiana University School of Medicine, Indianapolis, IN, USA; ${ }^{2}$ Department of Pulmonary, Critical Care, Sleep and Occupational Medicine, Indiana University School of Medicine, Indianapolis, IN, USA

Background: Pulmonary hypertension in heart failure with preserved ejection fraction $\mathrm{PH}-$ $\mathrm{HFpEF}$ ) is the most common cause of $\mathrm{PH}$ worldwide. It is closely linked to risk factors for metabolic syndrome, including obesity and diabetes - factors known to increase proliferation and migration of pulmonary artery smooth muscle cells (PASMCs), leading to pulmonary vascular remodeling. Qualitative studies have shown that patients with progressive vascular abnormalities develop more severe symptoms and suffer frequent hospitalization. However, underlying mechanisms involved in the regulation of pulmonary vascular remodeling in metabolic syndromeassociated PH-HFpEF are still unclear.

Aim: We have recently observed decreased levels of the tumor suppressor WW domaincontaining oxidoreductase (WWOX), which plays a housekeeping role in repressing cellular proliferation, in PASMCs isolated from rats with experimental PH-HFpEF and human subjects with obesity and diabetes. As microRNAs (miRNAs) have been shown to regulate WWOX expression in cancers, here we aimed at examining the involvement of miRNAs in WWOXassociated pulmonary vasculature regulation in metabolic syndrome-associated $\mathrm{PH}-\mathrm{HFpEF}$.

Methods and Results: Among miRNAs that have been associated with reduced WWOX expression, including miR -134-5p, -153-3p, -29a-3b, -29b-3p and -187-5p, we found that miR134-5p was significantly increased in PASMCs of obese and diabetic subjects. To determine the role of miR-134-5p in the regulation of WWOX in the pulmonary vasculature, we applied exogenous miR-134-5p to human PASMCs. Treatment with miR-134-5p decreased WWOX expression, increased PCNA expression (a cell proliferation marker) and enhanced cellular proliferation. Additionally, human PASMCs challenged with high concentration of glucose, palmitic acid and insulin, which mimic hyperglycemic, hyperlipidemic and hyperinsulinemic conditions, exhibited increased miR-134-5p, accompanied by elevated cellular proliferation.

Conclusions: These studies suggest that miR-134-5p may have a potential role in metabolic syndrome-associated PH-HFpEF through regulating WWOX in the pulmonary vasculature. These 
studies identify miR-134-5p as a potential therapeutic target for the treatment of metabolic syndrome-associated PH-HFpEF.

This project was funded, in part, with support from the NIH NHLBI Short-Term Training Program in Biomedical Sciences Grant funded, in part by T35HL110854 from the National Institutes of Health. The content is solely the responsibility of the authors and does not necessarily represent the official views of the National Institutes of Health. 